\title{
Les acides gras trans
}

\author{
Trans-fatty acids
}

\section{Saïd AZZOUG ${ }^{1,2}$, Djamila MESKINE ${ }^{1,2}$}

${ }^{1}$ Service d'Endocrinologie et Maladies Métaboliques EPH Bologhine Alger - Algérie.

${ }^{2}$ Laboratoire d'Endocrinologie et Métabolisme Université Alger 1 Alger - Algérie.

\section{Correspondance à :}

Said AZZOUG

saidazzoug@yahoo.fr

DOI:https://doi.org/10.48087/BJMS ra.2019.6105

Il s'agit d'un article en libre accès distribué selon les termes de la licence Creative Commons

Attribution International License (CC BY 4.0), qui autorise une utilisation, une distribution et une reproduction sans restriction sur tout support ou format, à condition que l'auteur original et la revue soient dûment crédités.

\begin{abstract}
RÉSUMÉ
Les études ont montré que la consommation des acides gras trans provenant de l'hydrogénation industrielle partielle des huiles végétales était néfaste pour la santé en augmentant notamment le risque cardiométabolique ; leur consommation devrait donc être limitée voir interdite comme le suggèrent certaines recommandations. Mais d'un autre côté, certains acides gras trans naturels issus des ruminants pourraient être bénéfiques pour la santé et leur consommation ne devrait de ce fait pas être restreinte. L'effet des acides gras trans devrait donc être nuancé en fonction de leur origine naturelle ou industrielle.
\end{abstract}

Mots-clés : Acides gras trans industriels, acides gras trans naturels, risque cardiovasculaire, risque métabolique.

\begin{abstract}
Consumption of trans-fatty acids derived from industrial partial hydrogenation of vegetable oils is a risk factor for metabolic syndrome, diabetes mellitus and coronary heart disease. Therefore, their consumption should be reduced or even eliminated as suggested by some recommendations. On the other hand, some ruminant specific trans-fatty acids have health benefits and their consumption should not be restricted. Effects of trans-fatty acids on health depend on their origin. While industrial ones are harmful, natural trans-fatty acids may be beneficial.
\end{abstract}

Keywords: Industrial trans-fatty acids, natural trans-fatty acids, cardiovascular risk, metabolic risk.

\section{Introduction}

Il est classiquement recommandé de limiter l'apport des acides gras dans le but de diminuer le poids des maladies cardiovasculaires et métaboliques. Les actions sur la consommation portent sur la quantité mais également sur la qualité des graisses. Il est notamment conseillé de limiter l'apport des acides gras saturés et des acides gras trans (AGT). Une consommation excessive d'AGT serait liée à une augmentation du risque des maladies cardiométaboliques. Cependant, l'effet des AGT devrait être nuancé en fonction de leur origine naturelle ou industrielle. En effet, certains AGT naturels pourraient avoir des effets bénéfiques.

\section{C'est quoi les acides gras trans?}

Les acides gras (AG) sont des chaines hydrocarbonées distinguées les unes des autres par la longueur de leur chaine carbonée, le degré d'insaturation ou la présence de doubles liaisons et la configuration spatiale de la ou des doubles liaisons.

Les AG sont classés en cis ou trans selon la configuration spatiale de la ou des doubles liaisons. Dans la configuration cis, les atomes d'hydrogène sont du même côté, tandis que dans la configuration trans les atomes d'hydrogène sont de part et d'autre de la double liaison [Figure 1]. Les AG sont dits trans (AGT) s'ils possèdent au moins une double liaison en configuration trans. La structure tridimensionnelle des AGT est ainsi similaire à celle des acides gras saturés. Les acides gras insaturés présents dans l'alimentation sont principalement de type cis, mais la configuration trans se rencontre également, sa proportion dépend du type d'aliments.

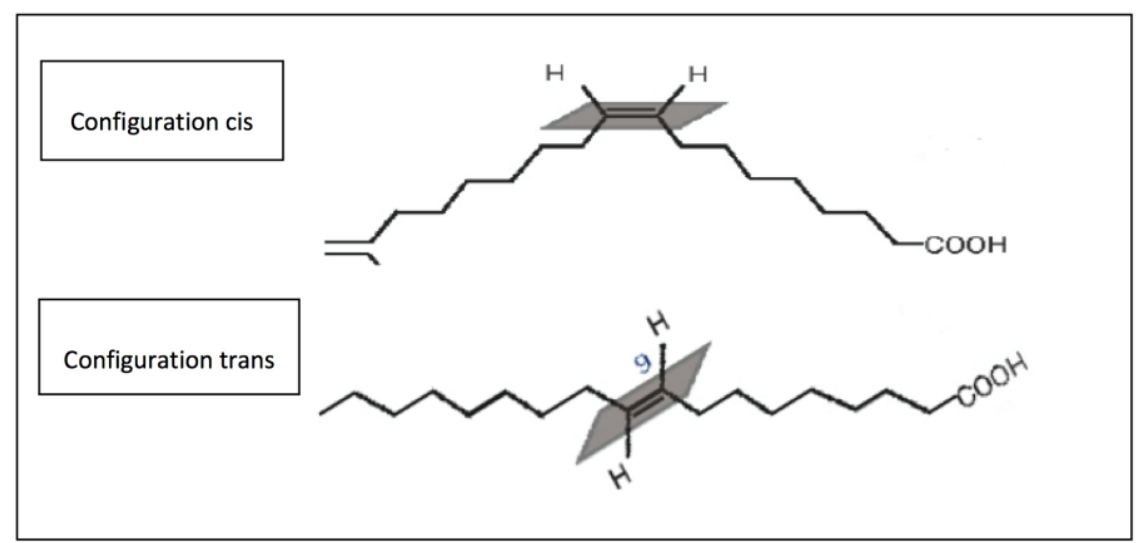

Figure 1. Configuration cis et trans. 


\section{D'où proviennent les AGT ?}

Les AGT proviennent de 3 sources :

- Origine naturelle : lait et dérivés et viandes des ruminants (bovins, ovins, caprins) et ceci suite à une transformation bactérienne des acides gras insaturés dans le rumen (premier estomac des ruminants).

- Hydrogénation industrielle: Procédés industriels d’hydrogénation partielle des graisses végétales. Le but principal de l'hydrogénation est de transformer les huiles végétales liquides en corps gras solides à température ambiante.

- Traitements thermiques: Chauffage à température élevée des graisses insaturées d'origine industrielle (raffinage/désodorisation des huiles végétales liquides) ou domestique (cuisson, fritures, grillades).

\section{Dans quels produits trouve-t-on les AGT?}

Pour l'industrie alimentaire, les huiles végétales partiellement hydrogénées sont attrayantes puisqu'elles sont moins chères que les graisses animales et ont des avantages commerciaux.

Elles ont une longue durée de vie, une bonne résistance à l'oxydation et aux températures de cuisson élevées. En outre, l'hydrogénation partielle améliore la consistance, le goût et la palatabilité des produits alimentaires. C'est un processus utilisé pour produire les margarines, les pates à tartiner et les shortenings (mélange de matières grasses anhydres destinées principalement à la biscuiterie).

Les produits alimentaires contenant ces graisses hydrogénées pourvoyeuses d'AGT sont les margarines, les produits de boulangerie pâtisserie (viennoiseries, cakes, cookies), confiserie (biscuits, barres chocolatées), les pizzas et les produits de fast food. La quantité d'AGT varie d'un produit à un autre. A titre d'exemple, le pourcentage d'AGT par rapport à la quantité totale d'acides gras dans les margarines peut varier de 1-2 \% dans les margarines de haute qualité à $60 \%$ dans les margarines de basse qualité [1].

\section{Quels seraient les risques des AGT?}

Les AGT sont incorporées dans les membranes cellulaires, ils peuvent ainsi altérer les propriétés physicochimiques des membranes cellulaires, influencer le fonctionnement et la signalisation des récepteurs membranaires et de différentes voies enzymatiques et hormonales. Ils peuvent également avoir une action génique vu que les acides gras sont des ligands naturels de multiples récepteurs cytosoliques qui régulent la transcription génique.

\section{AGT et maladies cardiovasculaires}

Il existe une relation positive entre une consommation élevée ( $>3$ gr par jour) d'AGT et le risque de maladies cardiovasculaires. Une consommation d'AGT correspondant à $2 \%$ de la dépense énergétique totale augmente le risque cardiovasculaire de $25 \%$. Les AGT seraient plus athérogènes que les acides gras saturés. C'est une des raisons qui ont fait que l'organisation mondiale de la santé (OMS) et plusieurs autres organismes de santé recommandent que l'apport d'AGT ne doive pas dépasser $1 \%$ de l'apport énergétique total.

Plusieurs mécanismes expliqueraient l'augmentation du risque cardiovasculaire par les AGT : dyslipidémie, insulino-résistance, stimulation des molécules pro-inflammatoires (interleukine 6, TNF $\alpha$, CRP), dysfonction endothéliale [2].

\section{AGT et risque métabolique}

Un apport élevé en AGT augmente le risque d'obésité viscérale, de syndrome métabolique, de dyslipidémie et de diabète de type 2.

Les AGT augmentent le risque d'obésité, notamment d'obésité viscérale. Le risque est plus important chez les sujets qui sont déjà obèses ou qui ont des antécédents familiaux d'obésité. Le risque de prise de poids est de trois à quatre fois plus important avec les AGT qu'avec les acides gras saturés.

Les AGT augmentent le risque d'insulinorésistance et de dysglycémies. Ils diminuent l'expression des GLUT4, la captation musculaire de glucose et la synthèse hépatique de glycogène. Ils entrainent également une diminution de l'expression des récepteurs de l'insuline au niveau hypothalamique.

Les AGT augmentent les taux de LDL cholestérol avec des LDL petites et denses et diminuent ceux du HDL cholestérol. Ils augmentent le nombre de particules Apo B et diminuent le nombre des Apo A. Par ailleurs, ils entrainent une augmentation des taux de triglycérides et de la lipoprotéine a $[3,4]$.

\section{AGT et cancers}

Des études ont montré qu'une consommation excessive d'AGT pourrait augmenter le risque de cancers notamment le cancer du sein et le cancer colorectal. Cependant, d'autres travaux n’ont pas confirmé ces données.

\section{AGT et risque tératogène}

Les AGT consommés par la mère traversent le placenta et sont sécrétés dans le lait maternel. Il existerait une corrélation inverse entre la consommation d'AGT et le poids de naissance. Par ailleurs, des taux élevés d'AGT et des taux bas d'oméga-3 dans le cordon ombilical affectent le développement neurologique et rétinien du bébé.

\section{Existe-t-il une différence entre les AGT naturels et les AGT industriels ?}

Les études qui ont rapporté des effets négatifs sur la santé ont concerné essentiellement les AGT industriels issus des graisses végétales partiellement hydrogénées. A l'inverse, des études récentes ont montré que certains AGT naturels pourraient avoir un effet bénéfique. 
Pour rappel, les AGT se présentent sous différentes formes chimiques en fonction de la longueur de la chaine carbonée, du nombre de doubles lésions, de la localisation et du nombre de doubles liaisons en position trans. Le type d'AGT diffère selon leur origine naturelle ou industrielle. Les isomères de l'acide oléique (18:1 c9) : l'acide vaccénique (18:1 t11) d'origine naturelle et l'acide élaïdique (18:1 t9) d'origine industrielle sont les principaux AGT.

Par ailleurs, les ruminants produisent outre l'acide vaccénique de petites quantités d'AGT polyinsaturés avec des doubles liaisons conjuguées comme les isomères de l'acide linoléique conjugué (CLA ou conjugated linoleic acid). L'acide ruménique $(18: 2 \mathrm{c} 9$, t11) est le principal isomère CLA. Ces CLA auraient des effets bénéfiques sur la composition corporelle, l'inflammation, l'immunité, le diabète et le cancer. Le lait et ses dérivés sont les principaux pourvoyeurs de ces CLA.

Les CLA améliorent la composition corporelle. Ils diminuent la masse grasse et augmentent la masse maigre. Ils améliorent également l'insulinorésistance et le profil lipidique. Par ailleurs, certains AGT naturels présents dans les dérivés laitiers diminueraient le risque de diabète de type 2. Les CLA ont un effet positif sur les réactions inflammatoires et la réponse immune par la modulation de la production de cytokines, de prostaglandines et d'immunoglobulines. Les CLA ont été utilisés dans les maladies inflammatoires intestinales avec des effets prometteurs. Les CLA diminuent la pression artérielle et améliorent la fonction endothéliale et la production des eicosanoïdes. Les CLA pourraient aussi avoir des effets anti-carcinogènes. Ils ont un effet antimutagène, ils inhibent la prolifération cellulaire et induisent l'apoptose [5].

\section{Qu'est - il recommandé concernant la consommation des AGT?}

La consommation moyenne d'AGT est de 1,3\% de l’apport énergétique total (AET). La consommation de 5\% de la population dépasse $2 \%$. Les adolescents ont la consommation la plus élevée. La consommation de $10 \%$ des garçons âgés entre 12 et 14 ans dépasse $2 \%$.

L'organisation mondiale de la santé recommande que la consommation d'AGT ne dépasse pas $1 \%$ de l'AET. Le contenu en AGT ne devrait pas dépasser $2 \%$ du total des graisses dans les huiles végétales et les margarines et $5 \%$ dans les autres aliments.

Il est conseillé de réduire l'apport des AGT industriels en limitant la consommation des aliments les plus pourvoyeurs de ces produits. Les produits concernés sont les viennoiseries, la pâtisserie, les barres chocolatées, les biscuits, les pizzas et les produits de panification [1].

\section{Conclusion}

Il est évident que les AGT industriels ont des effets négatifs sur la santé. Pour ce qui est des AGT naturels notamment les AGT conjugués, il est possible qu'ils aient des effets bénéfiques. De ce fait, si la consommation des AGT industriels doit être diminuée, celle des AGT naturels, notamment certains AGT naturels conjugués ou CLA ne devrait pas être restreinte.

Déclaration d'intérêts : les auteurs ne déclarent aucun conflit d'intérêt en rapport avec cet article.

\section{Références}

1. Menaa F, Menaa A, Menaa B, Treton J. Trans-fatty acids, dangerous bonds for health? A background review paper of their use, consumption, health implications and regulation in France. Eur J Nutr. 2013; 52:1289-1302.

2. Dawczynski C, Lorkowski S. Trans fatty acids and cardiovascular risk: does origin matter? Expert Rev Cardiovasc Ther. 2016 Sep;14(9):1001-5

3. Micha R, Mozaffarian D. Trans fatty acids: effects on metabolic syndrome, heart disease and diabetes. Nat Rev Endocrinol. 2009; 5: 335-344.

4. de Souza RJ, Mente A, Maroleanu A, Cozma Al, Ha V,Kishibe T, Uleryk E, et al. Intake of saturated and trans unsaturated fatty acids and risk of all cause mortality, cardiovascular disease, and type 2 diabetes: systematic review and meta-analysis of observational studies. BMJ. 2015;351:h3978.

5. Ferlay A, Bernard L, Meynadier A, Malpuech-Brugère $C$. Production of trans and conjugated fatty acids in dairy ruminants and their putative effects on human health: A review. Biochimie 2017 Oct;141:107-120.

Cet article a été publié dans le « Batna Journal of Medical Sciences » BJMS, l'organe officiel de « l'association de la Recherche Pharmaceutique - Batna»

Le contenu de la Revue est ouvert « Open Access » et permet au lecteur de télécharger, d'utiliser le contenu dans un but personnel ou d'enseignement, sans demander l'autorisation de l'éditeur/auteur.

Avantages à publier dans BJMS :

- Open access : une fois publié, votre article est disponible gratuitement au téléchargement

- Soumission gratuite : pas de frais de soumission, contrairement à la plupart des revues «Open Access »

- Possibilité de publier dans 3 langues : français, anglais, arabe

- Qualité de la relecture : des relecteurs/reviewers indépendants géographiquement, respectant l'anonymat, pour garantir la neutralité et la qualité des manuscrits.

Pour plus d'informations, contacter BatnaJMS@gmail.com ou connectez-vous sur le site de la revue : www.batnajms.net 